Palavras chave:

Análise de risco

Análise Econômica

Economia Florestal

Histórico:

Recebido 28/01/2013

Aceito 28/04/2015

Keyworks:

Risk Analysis

Economic Analysis

Forest Economy

Correspondence: maisajoaquim@unb.br

DOI:
Maísa Santos Joaquim', Álvaro Nogueira de Souza', Sandro Nogueira de Souza², Reginaldo Sérgio Pereira', Humberto Angelo'

\section{APLICAÇÃO DA TEORIA DAS OPÇÕES REAIS NA ANÁLISE DE INVESTIMENTOS EM SISTEMAS AGROFLORESTAIS}

RESUMO: Neste trabalho, compararam-se os valores gerados pela análise econômica tradicional (Valor Presente Líquido - VPL) com as Opções Reais. Aplicou-se o método proposto por Copeland \& Antikarov para opções arco-íris, devido às três variáveis estocásticas selecionadas, com decisão de abandono caso o valor do projeto com flexibilidade fosse menor que o valor do ativo subjacente. Possibilitando a geração do valor de flexibilidade para que o empreendedor tenha mais confiança na tomada de decisão no momento de investir em um projeto com Sistema Agroflorestal, com a visualização de diversos cenários em uma única análise. Os resultados demonstraram que a análise de viabilidade econômica com Opções Reais propicia a melhor visualização dos possíveis cenários, dentro de um intervalo gerado pela volatilidade dos preços. O VPL adotado como o valor do ativo subjacente foi de $\mathrm{R} \$ 5.684,32$, com a taxa de desconto (WACC) de $9,95 \%$ e taxa de juros livre de risco de 7,5\%. Para determinar a volatilidade calculou-se: 0 desvio padrão do retorno logaritmizado $(27,06)$, a média $(0,0883)$, valores dos movimentos ascendentes (u) I,3 I 07 com probabilidade de ocorrência de 6I,34\% e descendentes (d) 0,7628 com probabilidade de $38,66 \%$. O valor da opção em caso de abandono foi de $R \$$ $2.059,0 \mathrm{I} /$ ha positivo. Os resultados mostraram que o VPL superestimou o retorno do projeto e que seria viável abandoná-lo.

\section{THE THEORY OF REAL OPTIONS IN INVESTMENT ANALYSIS OF AGROFORESTRY SYSTEMS}

ABSTRACT: In this study we compared the values generated by traditional economic analysis (Net Present Value - NPV) with Real Options Method. The objective was to apply the method proposed by Copeland \& Antikarov for rainbow options, due to three stochastic variables selected with decision of abandonment if the project value with flexibility was less than the value of the underlying stock. It enabled the flexibility value generation in order to entrepreneurs to have more confidence in their decision when they are investing in a project with Agroforestry System, with different scenarios visualization in a single analysis. The results evidenced that the economic viability analysis, using Real Options, provided the best view of the possible scenarios, within a range generated by the prices volatility. The Net Present Value adopted as the underlying stock value was $\mathrm{R} \$$ $5,684.32$, using the discount rate (WACC) of $9.95 \%$ and risk-free interest rate of $7.5 \%$. To determine the volatility were calculated: the logarithmic return standard deviation (27.06), the average (0.0883), upward movements values (u) (I.3107) with $61.34 \%$ of probability and downward movements values (d)(0.7628) with $38.66 \%$ of probability. The option value in case of abandonment was $R \$ 2,059.01 /$ ha positive. The results showed that the NPV overestimated the project return and that it would be viable to abandon it.

' UNB, Brasília, Distrito Federal, Brasil

2 Faculdade Anhanguera, Valparaíso, Goiás, Brasil 


\section{INTRODUÇÃO}

O arranjo agrossilvipastoril vem se tornando um importante sistema de produção por ser considerado o sistema mais completo dentre os sistemas agroflorestais, atraindo produtores pelas inúmeras vantagens ecológicas e econômicas, focando a maximização do lucro (SANGUINO et al. 2007).

A existência de flexibilidades é constante em investimentos da área florestal como os sistemas agroflorestais, e, para essa classe, os métodos tradicionais de avaliação econômica de projetos utilizados podem não ser os mais indicados para avaliar corretamente o potencial de um projeto de investimento (RIES \& ANTUNES, 2007).

Para Oliveira (2009), antes da implantação de um consórcio comercial é ideal que haja um planejamento técnico e análise de viabilidade econômica prévia para que fiquem bem especificadas quais espécies comporão o sistema e as perspectivas de retorno do investimento com suas devidas volatilidades.

Os métodos tradicionais de avaliação econômica de projetos ignoram o valor associado à flexibilidade de se adiar as decisões sobre a implementação de um projeto. Para que se obtenha uma avaliação apropriada do valor das suas oportunidades de investimento em capital, deve-se utilizar a teoria do investimento sob incerteza, conhecida por Teoria das Opções Reais (TOR) (DAMODARAM, 2009).

Por definição, uma opção real é o direito, mas não a obrigação, de empreender uma ação (por exemplo, adiar, expandir, contrair ou abandonar ou investir em um projeto), a um custo predeterminado, denominado preço de exercício, por um período preestabelecido: a vida da opção (COPELAND \& ANTIKAROV, 2002).

Brandão et al. (2005) concluem que os métodos tradicionais de análise de investimentos como o Fluxo de Caixa Descontado (FCD) são incapazes de captar a volatilidade existente nos projetos agroflorestais e é neste cenário que a Teoria das Opções Reais mostra-se com maior eficiência.

Frey et al. (2009) afirmam que a modelagem pelas opções reais tornou-se muito importante e utilizada na economia florestal na última década e tem sido utilizada para estimar rotações ideais de colheita e regimes de desbaste pelo fato da colheita da madeira ser uma decisão flexível, podendo aguardar condições futuras ideais.

Para Sousa Neto et al. (2008), a opção real é uma ferramenta que auxilia a quantificar os riscos, por meio de uma perspectiva financeira, o valor de opções estratégicas. Segundo Cheng (2007), para existir uma opção real são necessárias três condições básicas: Irreversibilidade: o projeto é parcialmente ou totalmente irreversível, isto é, não é possível recuperar todo o investimento inicial caso haja mudança de planos; Incerteza: a respeito dos retornos futuros do investimento, o fluxo de caixa não é fixo e conhecido de antemão; Possibilidade de reavaliação: é possível postergar a ação para obter mais informações sobre o futuro.

Segundo Damodaran (2009), todas as técnicas existentes para avaliação de risco preconizam o risco de perdas e ignoram o componente da oportunidade no risco, e, as opções reais são as únicas que dão lugar de destaque para o potencial das oportunidades associado ao risco considerando a incerteza um valor adicional para aqueles que se preparam para tirar vantagens dela.

Mun (2002) afirma que a flexibilidade em exercer ou abandonar opções em diferentes períodos do fluxo de caixa, quando o grau de incerteza se dilui, é o que faz a teoria das opções reais tão valiosa para avaliar tomadas de decisão estratégicas e que essa flexibilidade gerencial torna as decisões mais realistas.

Segundo Dixit \& Pindyck (1994), a volatilidade é uma tentativa matemática de mensurar a incerteza em relação aos retornos proporcionados pela ação. De todos os fatores que alteram o preço da opção, a volatilidade é a mais subjetiva, e por isso, a mais difícil de medir e compreender.

A teoria das opções reais procura "mapear" todo conjunto de alternativas, e colocá-las dentro de uma árvore de decisão, analisando inclusive se o investimento, ou parte dele, é reversível e passível de ser abandonado. Depois, com o auxílio do método do valor presente líquido, fica fácil verificar a rentabilidade de cada uma das opções e decidir pela melhor, em cada parte do processo (BRASIL et al., 2007).

No Brasil e em outros países a TOR tem sido empregada para avaliar investimentos sob condições de incerteza, como mostrou DIAS (2005) que avaliou a exploração do petróleo e Castro (2000) o Setor Elétrico.

A análise tem por objetivo aplicar o método da Teoria das Opções Reais seguindo as etapas estabelecidas por Copeland \& Antikarov (2002). 


\section{MATERIAL E MÉTODOS}

\section{Área de estudo}

A área de estudo pertence à Votorantim Siderurgia, localizada no município de Vazante, na região Noroeste do estado de Minas Gerais. A latitude é 17³6'09" e a longitude é 46 42'02" Oeste de Greenwich. A altitude é de $550 \mathrm{~m}$. O clima é do tipo Aw, tropical úmido de savana, com inverno seco e verão chuvoso, segundo a classificação de Köppen (SOUZA et al., 2007). A temperatura média anual é de $24^{\circ} \mathrm{C}$ e a precipitação média anual é de $1.450 \mathrm{~mm}$.

As unidades de estudo foram compostas por talhões de um clone de híbridos naturais de Eucalyptus urophylla $\times$ Eucalyptus camaldulensis, plantados no espaçamento $9+3 \times 2$ metros, em consórcio com soja, implantada no espaço de 9 metros entre as linhas do eucalipto, em uma área útil de 6,4 metros nos anos I, 2 da primeira rotação, no ano 7 (após desbaste) e no ano 8 (final da primeira rotação) com produtividade de $18,67 \mathrm{sc} /$ ha (sacas por hectare) e boi gordo, em que o bezerro é introduzido no sistema para engorda a partir do ano 3, com peso médio de 8,25@ (saindo com 16,5@),com rotação anual, até o ano 6 e é reintroduzido a partir do ano 10 até 0 ano 14.

\section{Base de dados}

A base de dados utilizada na análise foi fornecida pela empresa sendo composta de informações de custos e receitas da implantação, condução e colheita dos produtos saca de soja e arroba (@) do boi gordo e, implantação e condução do eucalipto para venda do metro cúbico $\left(\mathrm{m}^{3}\right)$ da madeira em pé.

\section{Eucalipto}

O volume de madeira foi estimado via sistema próprio de prognose da produção. No primeiro desbaste, realizado no ano 6 , são retirados $75 \%$ do volume, que equivale a $120 \mathrm{~m}^{3}$, essa madeira é destinada a produção de energia.

No ano 8 , é realizado o corte raso da floresta e retira-se $120 \mathrm{~m}^{3}$, dos quais, $48 \mathrm{~m}^{3}$, equivalente a $40 \%$ do volume total é destinado ao uso nobre da madeira e, o restante, $60 \%\left(72 \mathrm{~m}^{3}\right)$, é utilizado para energia.
Após o corte raso, conduz-se a brotação das cepas, com expectativa de produtividade de $216 \mathrm{~m}^{3}$ ao final do ciclo, no ano 14. Toda madeira do segundo ciclo é destinada a energia. Os custos de implantação e manutenção estão descritos na Tabela I.

TABELA 1 Custos de implantação e manutenção do eucalipto no Sistema Agroflorestal.

TABLE 1 Planting and maintenance costs of eucalypt in Agroforestry System.

\begin{tabular}{ccccc}
\hline \multicolumn{5}{c}{ Implantação e Manutenção } \\
\hline $\begin{array}{c}\text { Operação } \\
\left(\mathrm{R} \$ \cdot h a^{-1}\right)\end{array}$ & Mecanizado & Manual & Insumos & $\begin{array}{c}\text { Custo } \\
\text { Total }\end{array}$ \\
\hline Total Geral & 712,85 & 346,10 & $1.216,89$ & $2.275,85$ \\
Total Geral & 808,90 & $1.965,07$ & $1.413,72$ & $4.187,69$ \\
\hline
\end{tabular}

Fonte: Votorantim Siderurgia (2012) adaptada pela autora.

\section{Método de Copeland \& Antikarov}

\section{O valor presente do projeto de SAF sem flexibilidade}

O valor presente do projeto de SAF sem flexibilidade foi calculado e avaliado, aplicando-se - método do Valor Presente Líquido (Rezende \& Oliveira, 2008) com fluxo de caixa anual. O horizonte de planejamento do projeto foi de 14 anos e taxa de 9,9\% a.a., calculado com WACC (equação I) por ter investimento de terceiros, sendo WACC ou CMPM = Retorno exigido $=T M A=$ Taxa de desconto; $R_{t}=$ Custo de capital de terceiros; $C T=$ TMA de terceiros; $R_{p}=$ Custo de capital da empresa; $C P=T M A$ da empresa.

$\mathbf{W A C C}=\left(\mathbf{R}_{\mathbf{t}} \cdot \mathbf{C T}\right)+\left(\mathbf{R}_{\mathbf{p}} \cdot \mathbf{C P}\right)$

A taxa de juros livre de risco utilizada foi a taxa média SELIC (Sistema Especial de Liquidação e Custódia) que registra as transações com títulos públicos e mede o custo de captação da dívida interna. De acordo com lubel (2008), essa taxa costuma ser adotada quando não existem ativos transacionados no mercado de capitais.

Para realização da análise, foi utilizada a taxa de desconto determinada pela empresa de 10\% a.a., associada à taxa de retorno exigida pelos investidores de $9,9 \%$ a.a., utilizando o WACC. Com a determinação do WACC, ajustou-se a nova Taxa Mínima Atratividade ponderada de $9,95 \%$ a.a. 
O valor do projeto (VPL) foi determinado descontando os fluxos de caixa futuros a uma taxa de desconto ajustada ao risco do projeto sem flexibilidade gerencial (equação 2), de acordo com Rezende \& Oliveira (2008), sendo: VPL = valor presente líquido; $R_{j}=$ receitas no período $j ; C_{j}=$ custos no período $j$; $W A C C=$ taxa de desconto; $j$ = período de ocorrência dos custos e das receitas; $n=$ número total de anos do fluxo de caixa.

$$
\mathrm{VPL}=\sum_{\mathbf{j}=0}^{\mathbf{n}} \mathbf{R}_{\mathbf{j}}\left(1+\mathbf{W A C C}^{-\mathbf{j}}-\sum_{\mathbf{j}=0}^{\mathbf{n}} \mathbf{C}_{\mathbf{j}}(1+\mathbf{W A C C})^{-\mathbf{j}}\right.
$$

$\mathrm{Na}$ realização dos cálculos, foi seguida as premissas do MAD (Marketed Asset Disclaimer), pois não existem projetos semelhantes negociados no mercado e o VPL é adotado como ativo subjacente no portfólio replicante (MÉNDEZ \& GOYANES, 2009).

\section{Construção da árvore de eventos}

A Construção da árvore de eventos (a árvore de eventos não incorpora decisões) partindo-se de $t_{0}$ até $t_{n}$, com o objetivo de modelar as incertezas que influenciam o valor do ativo subjacente sujeito a risco ao longo do tempo.

O processo estocástico dos preços é dado pelo movimento geométrico browniano (SAMUELSON, 1965), conforme equação 3 , sendo: $P=$ preço do ativo no instante $t ; \mu=$ taxa de crescimento de $P$ (drift); $\sigma=$ volatilidade de $P$ (desvio-padrão instantâneo); $d z=$ incremento de um processo de Wiener (incremento do caminho aleatório da variável selecionada, com distribuição normal e tempo definido).

$\mathrm{dP}=\mu \mathrm{Pdt}+\sigma \mathrm{Pd} z$

Para simplificar o cálculo foi utilizado o modelo binomial de Cox, Ross \& Rubinstein (1979). Neste modelo, o processo estocástico contínuo do preço do ativo descrito pela equação (5) é substituído por um caminho aleatório discreto com dois estados u (up) e d (down), que correspondem a uma alta e uma baixa do preço, considerando a probabilidade de ocorrência e a volatilidade.

A volatilidade (desvio padrão anualizado da distribuição dos retornos de um projeto em condições de incerteza) do valor agregado do projeto foi calculada utilizando as equações 4, 5 e 6, sendo: $z=$ Variável de previsão; $n$ n = logaritmo neperiano; $V P_{1}=$ Valor presente em $t_{1} ; V P_{0}=$ Valor presente em $t_{0} ; F C_{1}=$ Fluxo de caixa em $t_{1}$.

$\mathrm{z}=\ln \left(\frac{\mathrm{VP}_{1}+\mathrm{FC}_{1}}{\mathrm{VP}_{0}}\right)$

$V P_{0}=\sum_{t=1}^{T} \frac{F C_{t}}{(1+W A C C)^{t}}$

$\mathrm{VP}_{1}=\sum_{\mathrm{t}=2}^{\mathrm{T}} \frac{\mathrm{FC}_{\mathrm{t}}}{(1+\mathrm{WACC})^{\mathrm{t}-1}}$

Com esses dados foi realizada uma simulação utilizando o método de Monte Carlo, para a obtenção da nova volatilidade (incerteza) que foi utilizada como única em todo projeto, ano a ano, e aplicada para elaboração da árvore de eventos binomial.

\section{Decisões gerenciais}

Determinaram-se decisões gerenciais tomadas nos nós da árvore de evento, para transformá-la em árvore de decisões binomial replicante, utilizando probabilidades neutras ao risco. Os valores prevêem os movimentos ascendentes $u$ e descendentes $d$ e suas probabilidades neutras a risco $p$ e 1-p, taxa de juros livre de risco $\left(R_{f}\right)$, volatilidade do projeto $(\sigma)$, variação do tempo $(\Delta \mathrm{t})$, conforme as equações $7,8,9,10,11$ e 12 , sendo $u=$ movimento ascendente - valor que multiplica o projeto com uma evolução positiva e será sempre maior que 1 ; $d=$ movimento descendente assumido no momento 1 do projeto e será sempre menor que $1 ; \sigma=$ volatilidade do projeto; $\mathrm{e}^{\sigma}=$ intervalo de tempo limitado a $1 ; p=$ probabilidade neutra ao risco (risco-neutro).

$\mathrm{u}=\mathrm{e}^{\sigma \sqrt{\Delta t}}$

$\mathrm{d}=\frac{1}{\mathrm{u}}$ ou $\mathrm{d}=\mathrm{e}^{-\sqrt[\sigma]{\Delta t}}$

$\mathrm{FCn}$ ascendente $=\mathrm{FC}_{\mathrm{n}-1} \cdot \mathrm{u}$

$\mathrm{FCn}$ descendente $=\mathrm{FC}_{\mathrm{n}-1} \cdot \mathrm{d}$

$p=\frac{\left(1+R_{f}\right)-d}{(u-d)}$

$q=(1-p)$

\section{Cálculo da opção}

O cálculo foi realizado de trás (últimos períodos) para frente (até o primeiro período). No último período, o valor do fluxo de caixa foi o máximo entre o valor da opção e zero, assim, para cada célula da última coluna a 
opção foi avaliada conforme equação 13 , sendo $V_{O R}=$ valor da opção real; $V=$ valor presente da árvore de eventos e $X=$ preço de exercício da opção.

$\mathrm{V}_{\mathrm{OR}}=\operatorname{Max}[$ Vuoud $-\mathrm{X} ; 0]$

A equação 13 foi aplicada tanto para $u$ quanto

para d. Quando o valor presente do projeto calculado no nó correspondente da árvore de eventos for maior que o valor investido, a opção deverá ser exercida e seu valor é V - X. Caso contrário, a opção não deverá ser exercida e seu valor é zero.

Após o cálculo do valor da opção, a árvore de decisão foi montada com os valores obtidos para visualizar as decisões a serem tomadas. A abordagem da análise por árvore de decisões para o orçamento de capital pode levar em consideração decisões futuras feitas pela empresa e incorporar alguma flexibilidade administrativa no projeto.

O VPL expandido (VPL + o valor da opção) é expresso pela equação 14, sendo: $\mathrm{VPL}_{\text {exp }}=$ Valor Presente Líquido expandido; $\mathrm{VPL}_{\mathrm{T}}=$ Valor Presente Líquido tradicional; $\mathrm{VPL}_{\mathrm{OR}}=$ Valor Presente da opção.

$\mathrm{VPL}_{\exp }=\mathrm{VPL}_{\mathrm{T}}+\mathrm{VPL}_{\mathrm{OR}}$

\section{RESULTADOS E DISCUSSÃO}

O cenário apresentado com a avaliação de viabilidade econômica tradicional está em concordância com vários trabalhos que analisaram Sistemas Agroflorestais, como, Souza (2007), Coelho Júnior et al. (2008), Cordeiro (20I0), Müller et al. (20I I), com os projetos apresentando retorno ótimo, em média, no sexto ano, quando ocorre o primeiro corte da madeira.

$O$ valor adotado como valor do ativo subjacente sujeito a risco foi o valor presente líquido (VPL) de R\$ $5.684,32 / \mathrm{ha}$, do $14^{\circ}$ ano. Esse valor serviu de comparação com os outros valores gerados nas árvores de decisão.

Foi, então, determinada a frequência de ocorrência dos valores para composição do histograma apresentado na Figura I, a média de 0,8836 e o desvio padrão de 27,06\% para ser utilizado nos cálculos. Os valores devem ter, obrigatoriamente, distribuição normal, premissa básica para que se possa efetuar a análise utilizando opções reais (BRASIL et al.,2007).

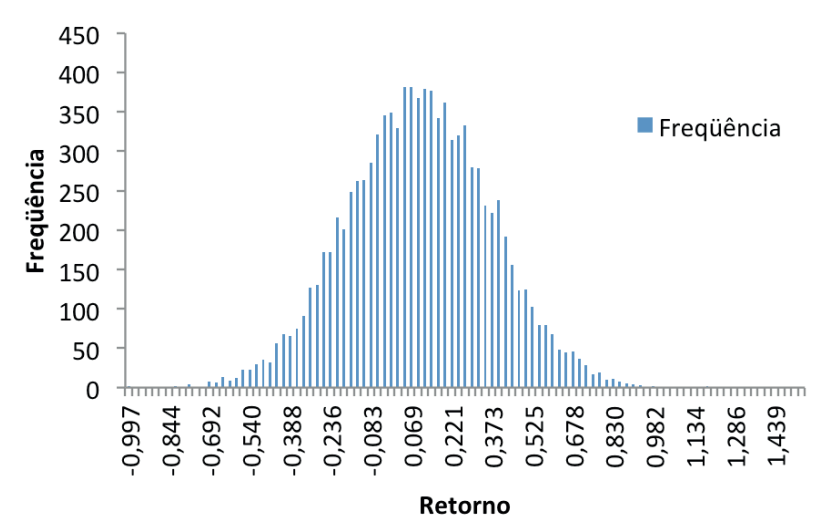

FIGURA 1 Histograma de frequência do VPL. FIGURE 1 Histogram NPV frequency.

A probabilidade de o movimento ascendente acontecer é de $61,34 \%$ e do movimento descendente é de $38,66 \%$. A árvore de eventos está apresentada na Figura 2.

Conforme explícito na árvore de eventos, em condições totalmente propícias em que o valor presente do projeto atingisse o ápice da probabilidade e da volatilidade, o retorno do projeto pode chegar, no $14^{\circ}$ ano, a R\$25I.270,25/ha. Tal valor está absolutamente em desacordo com a realidade dos projetos Agroflorestais.

Os projetos estudados por Souza Neto, Oliveira \& Bergamini Júnior (2008), também apresentaram valores irreais para os devidos setores analisados. No entanto, os valores possíveis de ocorrer seguem o movimento de reversão à média e sempre ocorrem em torno da média, nunca alcançando os pontos extremos da árvore (CORREIA NETO, 2009). Esses trabalhos corroboram com os resultados alcançados na construção da árvore de eventos do projeto em questão.

A disposição binomial dos valores na árvore de eventos impossibilita a visualização dos demais valores possíveis de ocorrer dentro do intervalo gerado entre o mínimo e o máximo da variável selecionada.

O valor de abandono foi determinado somando-se todos os custos dispendidos para implantação do sistema e descontados à taxa utilizada para calcular o Valor Presente Líquido, 9,9\%, observado na Tabela 2. 
TABELA 2 Valor do Abandono.

TABLE 2 Renunciation Value.

\begin{tabular}{cccc}
\hline Ano & Custos Anuais $(\mathrm{R} \$)$ & Custos Anuais Acumulados (R\$) & Valor Presente dos Custos (R\$) \\
\hline 0 & $3.126,58$ & $3.126,58$ & $3.126,58$ \\
1 & $1.281,15$ & $4.407,73$ & $4.292,32$ \\
2 & $1.102,52$ & $5.510,25$ & $5.205,15$ \\
3 & $1.019,68$ & $6.529,92$ & $5.973,34$ \\
4 & 799,44 & $7.329,37$ & $6.521,36$ \\
5 & 869,79 & $8.199,15$ & $7.063,89$ \\
6 & 963,11 & $9.162,26$ & $7.610,52$ \\
7 & $1.485,11$ & $10.647,38$ & $8.377,48$ \\
8 & $1.041,86$ & $11.689,23$ & $8.867,07$ \\
9 & 849,58 & $12.538,81$ & $9.230,33$ \\
10 & 907,26 & $13.446,07$ & $9.583,31$ \\
11 & 869,79 & $14.315,86$ & $9.891,24$ \\
12 & 869,79 & $15.185,64$ & $10.171,42$ \\
13 & 869,79 & $16.055,43$ & $10.426,36$ \\
14 & 944,73 & $17.000,17$ & $10.678,33$ \\
\hline
\end{tabular}

\begin{tabular}{|c|c|c|c|c|c|c|c|c|c|c|c|c|c|c|}
\hline 0 & 1 & 2 & 3 & 4 & 5 & 6 & 7 & 8 & 9 & 10 & 11 & 12 & 13 & 14 \\
\hline & & & & & & & & & & & & & & $251.270,25$ \\
\hline & & & & & & & & & & & & \multicolumn{3}{|c|}{$191.693,79$} \\
\hline & & & & & & & & & & & & \multicolumn{2}{|l|}{$146.242,97$} & \multirow[t]{2}{*}{$146.242,9$} \\
\hline & & & & & & & & & & & $111.568,59$ & & $111.568,59$ & \\
\hline & & & & & & & & & & $85.115,55$ & & $85.115,55$ & & \multirow[t]{2}{*}{$85.115,55$} \\
\hline & & & & & & & & & $64.934,56$ & & $64.934,56$ & & $64.934,56$ & \\
\hline & & & & & & & & $49.538,50$ & & $49.538,50$ & & $49.538,50$ & & \multirow[t]{2}{*}{$49.538,50$} \\
\hline & & & & & & & $37.792,86$ & & $37.792,86$ & & $37.792,86$ & & $37.792,86$ & \\
\hline & & & & & & $28.832,13$ & & $28.832,13$ & & $28.832,13$ & & $28.832,13$ & & \multirow[t]{2}{*}{$28.832,13$} \\
\hline & & & & & $21.996,00$ & & $21.996,00$ & & $21.996,00$ & & $21.996,00$ & & $21.996,00$ & \\
\hline & & & & $16.780,72$ & & $16.780,72$ & & $16.780,72$ & & $16.780,72$ & & $16.780,72$ & & \multirow[t]{2}{*}{$16.780,72$} \\
\hline & & & $12.802,00$ & & $12.802,00$ & & $12.802,00$ & & $12.802,00$ & & $12.802,00$ & & $12.802,00$ & \\
\hline & & $9.766,63$ & & $9.766,63$ & & $9.766,63$ & & $9.766,63$ & & $9.766,63$ & & $9.766,63$ & & $9.766,63$ \\
\hline & $7.450,95$ & & $7.450,95$ & & $7.450,95$ & & $7.450,95$ & & $7.450,95$ & & $7.450,95$ & & $7.450,95$ & \\
\hline \multirow[t]{14}{*}{$\underline{5.684 .32}$} & & $5.684,32$ & & $5.684,32$ & & $5.684,32$ & & $5.684,32$ & & $\underline{5.684,32}$ & & $\underline{5.684,32}$ & & $\underline{5.684,32}$ \\
\hline & $4.336,56$ & & $4.336,56$ & & $4.336,56$ & & $4.336,56$ & & $4.336,56$ & & $4.336,56$ & & $4.336,56$ & \\
\hline & & $3.308,36$ & & $3.308,36$ & & $3.308,36$ & & $3.308,36$ & & $3.308,36$ & & $3.308,36$ & & $3.308,36$ \\
\hline & & & $2.523,94$ & & $2.523,94$ & & $2.523,94$ & & $2.523,94$ & & $2.523,94$ & & $2.523,94$ & \\
\hline & & & & $1.925,51$ & & $1.925,51$ & & $1.925,51$ & & $1.925,51$ & & $1.925,51$ & & $1.925,51$ \\
\hline & & & & & $1.468,97$ & & $1.468,97$ & & $1.468,97$ & & $1.468,97$ & & $1.468,97$ & \\
\hline & & & & & & $1.120,68$ & & $1.120,68$ & & $1.120,68$ & & $1.120,68$ & & $1.120,68$ \\
\hline & & & & & & & 854,96 & & 854,96 & & 854,96 & & 854,96 & \\
\hline & & & & & & & & 652,25 & & 652,25 & & 652,25 & & 652,25 \\
\hline & & & & & & & & & 497,60 & & 497,60 & & 497,60 & \\
\hline & & & & & & & & & & 379,62 & & 379,62 & & 379,62 \\
\hline & & & & & & & & & & & 289,61 & & 289,61 & \\
\hline & & & & & & & & & & & & 220,94 & & 220,94 \\
\hline & & & & & & & & & & & & & 168,56 & \\
\hline
\end{tabular}

FIGURA 2 Árvore de eventos baseada nos VPLs.

FIGURE 2 Events tree based on NPVs.

Os valores gerados foram comparados, nó a nó, com a árvore de decisão gerada, em que os valores dos últimos nós, no último ano $\left(14^{\circ}\right)$ foram calculados utilizando a taxa de juros livre de risco - SELIC - 7,5\%, conforme demonstrado Figura 3, árvore de decisão. 


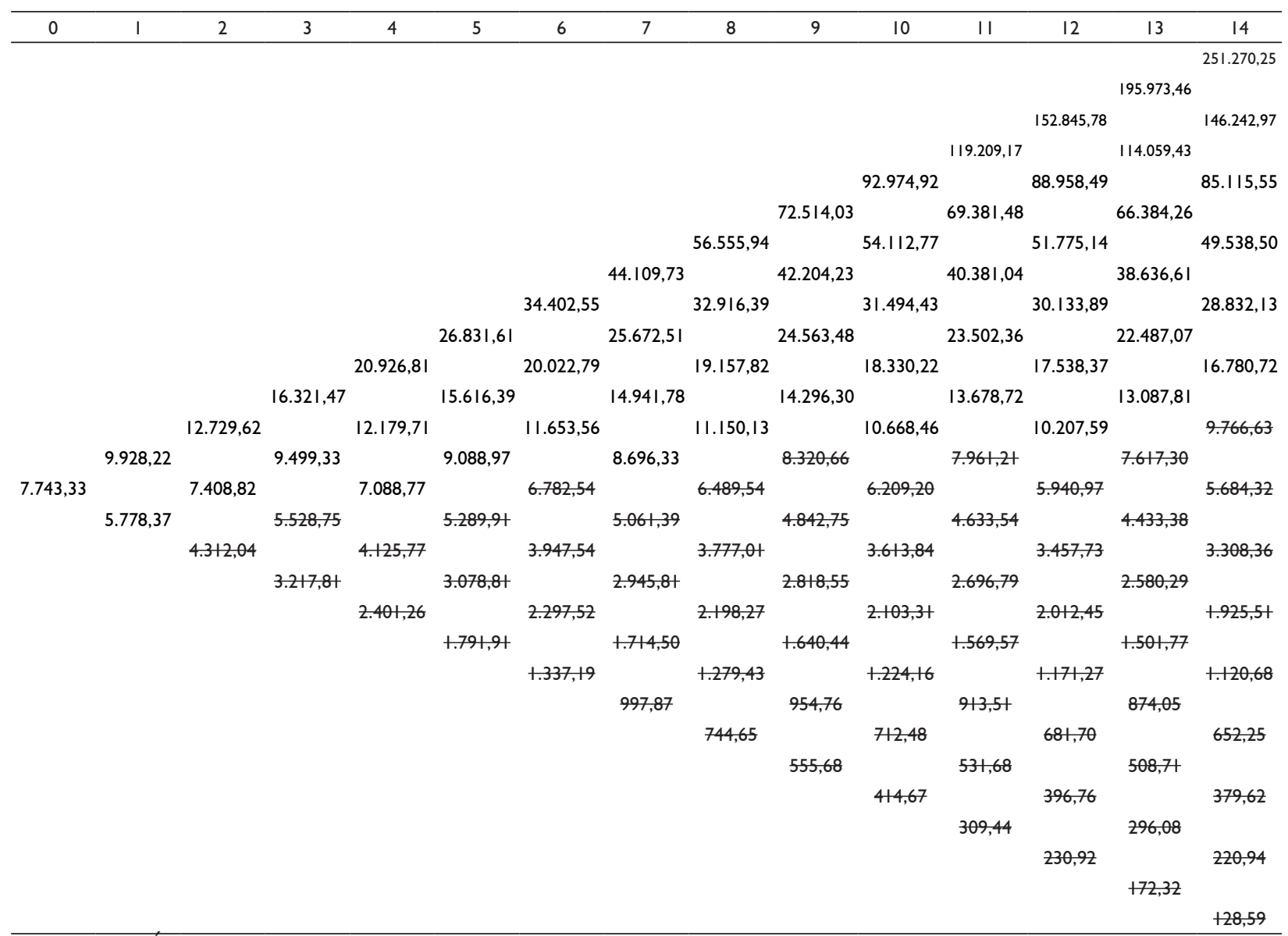

FIGURA 3 Árvore de Decisão com Taxa de Juros Livre de Risco.

FIGURE 3 Decision Tree with Risk-free Interest Rate.

O valor da opção para essa análise foi de $R \$$ $2.059,01 /$ ha positivos. Isso quer dizer que em caso de abandono, o investidor obterá lucro de $\mathrm{R} \$ 2.059,01$. Esse valor foi obtido ao se inserir a flexibilidade e a probabilidade de ocorrência da volatilidade dos possíveis retornos do projeto (VPL). Com isso, o projeto será mais viável economicamente se for vendido.

Tal fato fere a premissa essencial para aplicação da TOR, a volatilidade, pois, se os valores definidos como variáveis estocásticas permanecem fixos ao longo de todo o projeto é impossível determinar sua oscilação no horizonte de planejamento.

Percebeu-se com isso que, a volatilidade determinada com o Valor Presente Líquido, captou e combinou apenas, dois preços da madeira, um preço da soja, um preço da arroba do boi, produtividade do eucalipto e a taxa de juros que descapitalizou o Fluxo de Caixa.

Essa percepção ficou evidente quando se construiu a árvore de decisão utilizando ao invés da taxa livre de risco $(7,5 \%)$, a taxa mínima de atratividade do projeto $(9,9 \%)$. Com isso, os valores não sofreram alteração. Sob essa ótica é indiferente à utilização da TOR ou do VPL.

\section{CONCLUSÕES}

A aplicação do Método seguindo as quatro etapas estabelecidas por Copeland \& Antikarov (2002) é plausível, desde que algumas adaptações sejam realizadas para suprir as peculiaridades dos Projetos de SAF.

Para a realização da análise da viabilidade econômica em sistemas agroflorestais, nesse caso específico, a utilização das opções reais ou do valor presente líquido, é indiferente, pois o resultado não apresentou nenhuma diferença.

Para que se possa aplicar o método de opções reais na respectiva análise, faz-se necessário a utilização de séries históricas das variáveis selecionas, podendo assim, determinar os valores dos critérios básicos exigidos. 


\section{REFERÊNCIAS}

BRANDÃO, L.E.T.; DYER, J.S.; HAHN, W.J.. Using Binomial Decision Trees to Solve Real Option Valuation Problems. Decision Analysis, Vol.0, No.0, p. I-20, 2005.

BRASIL, H. G., FREITAS, J. M., DE, MARTINS, V. I. O., GONÇALVES, D. S., RIBEIRO, E. Opções Reais: Conceitos e Aplicações a Empresas e Negócios. Editora Saraiva, São Paulo - SP, 2007. ISBN 978-0206057-9.

CASTRO, A. L. Avaliação de Investimento de Capital em Projetos de Geração Termoelétrica no Setor Elétrico Brasileiro Usando Teoria das Opções Reais, Dissertação de Mestrado, Dep. de Eng. Ind., PUC/ Rio, Abril de 2000, 106p;

CHENG, Y. J., Decisão de Investimento através da Teoria de Opções Reais: Estudo de Caso em Projetos do Setor Financeiro. FGV, Dissertação de Mestrado, São Paulo, 2007.

COELHO JÚNIOR, L. M., REZENDE, J. L. P. DE, OLIVEIRA, A. D. DE, COIMBRA, L. A. B., SOUZA, A. N. de. Análise de Investimento de um Sistema Agroflorestal sob Situação de Risco. Revista Cerne, Lavras - MG, v. I4, n.4, p.368 378, outubro/dezembro 2008

COPELAND, T. E.; ANTIKAROV, V., Opções Reais: Um Novo Paradigma para Reinventar a Avaliação de Investimentos, Rio de Janeiro, Campus, 2002.

CORDEIRO, S. A. Avaliação Econômica e Simulação em Sistemas Agroflorestais. Tese de Doutorado em Ciências Florestais, Universidade de Viçosa - UFV, dezembro de 2010, pág. 85 .

CORREIO NETO, J. F. Elaboração e Avaliação de Projetos de Investimento: Considerando o Risco. Rio de Janeiro - RJ, Elsevier, Editora Campus, 20I0. ISBN 978-85-3523644-6.

COX, J.; ROSS, S.; RUBINSTEIN, M., Option pricing: A simplified approach. Journal Financial Economic. N. 7, p. $229-263,1979$.

DAMODARAN, A. Avaliação de Investimentos. Rio de Janeiro: Qualimark, 2009.

DIAS, M. A. G. Opções Reais Híbridas com Aplicação em Petróleo. 2005. Tese (Doutorado em Engenharia de Produção) - Pontifícia Católica do Rio de Janeiro (PUC), Rio de Janeiro, 2005.
DIXIT, A. K., PINDYCK, R. S., Investment under Uncertainty. New Jersey, Princeton University Press, 1994.

IUBEL, F. B. Análise das Decisões de Investimentos de um Plano de Saúde a partir da Teoria das Opções Reais. Dissertação de mestrado - Pontifícia Universidade Católica do Paraná, Curitiba - PR, 2008.

FREY, G. E., MERCER, D. E., CUBBAGE, F. W., ABT, R. C. A Real Options Method for Estimating the Adoption Potential of Forestry and Agroforestry Systems on Private Lands in the Lower Mississippi Alluvial Valley, USA. XII World Forestey Congress, Buenos Aires, Argentina, 18 - 23 October, 2009.

MENDÉZ, M.; GOYANES, A. Real Options Valuation of a Wind Farm. ESIC Business and Marketing School, Saphire Finance LLP. Business Finance Department, Universidad Autónoma de Madrid, February, 2009.

MUN, J., Real Options Analysis: Tools and Techniques for Valuing Strategic Investments and Decisions; Hoboken, New Jersey, John Wiley \& Sons, Inc., 2002.

MÜlleR, M. D., NOGUEIRA, G. S., CASTRO, C. R. T. DE, PACIUllo, D. S. C., ALVES, F. F., CASTRO, R. V. O., FERNANDES, E. N. Economic Analysis of na Agrosilvipastoral System for a Mountainous area in Zona da Mata Mineira, Brazil. Pesquisa Agropecuária Brasileira, Brasília - DF, v.46, n.10, p. I I48 - II53, outubro 201 I.

OLIVEIRA, T. C. de, Caracterização, Índices Técnicos e Indicadores de Viabilidade Financeira de Consórcios Agroflorestais. Dissertação de Mestrado em Produção Vegetal, Universidade Federal do Acre - UFAC, Rio Branco - AC, 83 f., 2009.

REZENDE, J. L. P.; OLIVEIRA, A. D. Análise Econômica e Social de Projetos Florestais. Viçosa: Universidade Federal Viçosa, 389 p. 2008.

RIES, L. R.; ANTUNES, L. M. Comercialização Agropecuária: Mercado Futuro e de Opções. Livraria e Editora Agropecuária, Guaíba - RS, I42p. ISBN 85-85437-60-0, 2007.

SAMUELSON, P. Proof that Properly Anticipated Prices Fluctuate Randomly. Industrial Management Review. p. $4 I$ - 49, Spring, 1965

SANGUINO, A. C.; SANTANA, A. C. DE; HOMMA, A. K. O.; BARROS, P. L. C. DE; KATO, O. K.; AMIN, M. M. G. H. Análise Econômica de Investimentos em Sistemas de Produção Agroflorestal no Estado do Pará. Revista Ciências Agrárias, Belém, n. 47, p. 23-47, jan/jun. 2007. 
SOUSA NETO, J. A., DE, OLIVEIRA, V. I., BERGAMINI JUNIOR, L. C. Opções Reais: Introdução à Teoria e à Prática. Qualitymark, Rio de Janeiro - RJ, 2008. ISBN 97885-7303-763-0.
SOUZA, A. N. DE, OLIVEIRA, A. D. DE, SCOLFORO, J. R. S., REZENDE, J. L. P. DE, MELLO, J. M. Viabilidade Econômica de um Sistema Agroflorestal. Cerne, v. 13, no.I, p.96-106, Lavras - MG, jan./mar. 2007. 